\title{
Henri Justino \\ The ALARA concept in pediatric cardiac catheterization: techniques and tactics for managing radiation dose
}

\begin{abstract}
The cardiac catheterization laboratory plays an important role in the management of children with congenital heart disease by not only enabling diagnosis but, in many cases, providing definitive therapy. The goal of the ALARA (As Low as Reasonably Achievable) concept as it applies to cardiac catheterization is to provide maximal diagnostic and therapeutic benefit while requiring the lowest possible radiation dose. A number of specific challenges unique to the setting of pediatric cardiac catheterization, such as higher heart rates, smaller cardiovascular structures, smaller body size, and wider variety of unusual anatomic variants with the potential need for relatively lengthy and complex studies, result in relatively high radiation doses (to the patient and, consequently, to laboratory personnel). In addition, the improved survival of patients with complex anatomy (e.g., palliated single ventricle anatomies) implies that many such children with chronic cardiac disease require frequent catheterizations within the first few years of life. These factors, coupled with the increased radiosensitivity of children and a longer lifespan ahead of them in which to possibly develop radiation-related sequelae, converge to create potentially ominous consequences. Attention to basic rules of radiation safety is, therefore, of tremendous importance in the pediatric cardiac catheterization laboratory. This review focuses on the importance of adequate planning of the study, optimizing image formation, management of fluoroscopy and cine angiography parameters, and the use of certain equipment features that might allow the cardiologist to lower the radiation dose without sacrificing image quality.
\end{abstract}

\footnotetext{
H. Justino $(\bowtie)$

The Charles E. Mullins Cardiac Catheterization Laboratories,

The Lillie Frank Abercrombie Section of Pediatric Cardiology, Department of Pediatrics,

Texas Children's Hospital,

Baylor College of Medicine,

6621 Fannin, MC 19345-C,

Houston, TX 77030, USA

e-mail: hjustino@bcm.edu

Tel.: +1-832-8265662

Fax: $+1-832-8255923$
}

Keywords Cardiac catheterization - Radiation exposure · Technical parameters

\section{Introduction}

The principles of radiation safety have been thoroughly expounded in excellent textbooks $[1,2]$ and journal articles $[3,4]$. Why another review article on this topic? First, the importance of safe radiation practice during cardiac catheterization cannot be overstated. While echocardiography and MRI have played increasingly important roles in the management of children with congenital heart disease, cardiac catheterization still possesses unique attributes that other imaging modalities have yet to match or exceed. As long as ionizing radiation is being utilized during cardiac catheterization, and it appears that this will be the case for the foreseeable future, emphasis on the teaching of techniques for lowering radiation dose will need to be made to trainees in pediatric cardiology. Second, there is no known "safe dose" of radiation, whether for the patient, the physician, or the staff in the cardiac catheterization laboratory. Therefore, the lower the radiation dose, the better. Third, in comparison to interventional radiologists, interventional cardiologists receive only a small fraction of the training in the areas of radiation physics, radiation biology, principles of image formation, and radiation safety. Fourth, there are specific challenges inherent to safe use of radiation while performing cardiac catheterizations on children, yet those challenges are rarely discussed because of the highly specialized nature of this field.

Children undergoing cardiac catheterization are potentially at greater risk of radiation-induced stochastic injuries resulting from the greater radiation sensitivity of their tissues compared with those of adults (radiation sensitivity is related to the rate of cell division in a given tissue), along with the longer lifespan ahead of them in which to develop potential neoplasms. Those children with complex cardiac anomalies are at greater risk because of the need for frequent cardiac catheterizations during childhood, coupled with the increased duration (and radiation burden) of 
complex interventional procedures. Furthermore, some of the tissues sensitive to radiation injuries (the eyes, thyroid, and gonads) are significantly closer to the heart in young children than in adults, placing them in closer proximity to scattered rays and to the primary beam. Smaller patient size and higher heart rate also result in the greater need for magnification modes and higher frame rates, all of which contribute to a significant increment in radiation dose. The smaller patient size means greater proximity of the operator to the primary beam and to the scattered rays. On a salutary note, smaller patient size means a lower radiation dose to achieve adequate image density and less scatter; less scatter in turn reduces the radiation exposure to the operator and personnel and improves image contrast. The smaller the child, the farther the skin is from the X-ray source, which also aids in reducing radiation doses.

There exists an intrinsic compromise between radiation dose and image quality. A discussion focused solely on lowering radiation dose (without attention to image quality) would have the operator reducing radiation levels to an absolute minimum; this approach could potentially significantly compromise image quality, and ultimately patient care. Similarly, optimizing image quality alone without attention to radiation dose would potentially result in unacceptably elevated radiation doses, which would also be detrimental to the patient. Thus, no clinically relevant discussion of radiation safety should exclude the teaching of proper imaging techniques: the two competing aims are inextricably linked. A practical approach to the practice of pediatric cardiac catheterization as it pertains to radiation safety and image quality is presented here. We begin with an overview of the basic functioning and layout of cardiac catheterization laboratory equipment and follow with a discussion of how X-ray beam characteristics affect image quality and radiation dose. The final part is a list of 20 practical tips for the proper planning of angiographic studies, for optimizing image quality, and for minimizing radiation exposure. Some of the tactics discussed might not appear at first glance to be of benefit in reducing radiation dose; however, the relevance of these points becomes clear when they are taken within the larger context of aiming to achieve excellence in image quality while simultaneously minimizing the radiation dose.

\section{Cardiac catheterization laboratory equipment overview}

Most pediatric cardiac catheterization laboratories are equipped with biplane fluoroscopy units. Although many of the concepts discussed herein apply regardless of the type of image receptor used, for the purposes of illustrating principles of radiation safety, units equipped with image intensifiers (IIs) are discussed. The newer flat panel detectors are discussed elsewhere in this monograph.

Biplane laboratories are set up with the frontal II positioned anterior to the patient with the corresponding $\mathrm{X}$-ray tube located posterior to the patient. Therefore, the $\mathrm{X}$-rays enter through the patient's back and exit through the patient's chest. A second gantry perpendicular to the first is typically arranged with the X-ray tube located to the patient's right (i.e. on the operator's side), and the II located to the patient's left. The lateral gantry arrangements are chosen by convention, in order to minimize magnification of the heart (the II is positioned on the side closest to the heart, i.e. leftward). Although this convention is derived from radiology practice designed to minimize artifactual enlargement of the heart from magnification, in real life this has little impact on the practice of cardiology, where the appearance of the heart size on fluoroscopy has little bearing on clinical decisions made in the catheterization laboratory. In fact, in the pediatric population, angiograms are as likely to involve left-sided structures as they are right-sided structures (e.g., this standard gantry arrangement reduces the magnification of the left pulmonary artery but maximizes magnification of the right pulmonary artery). As we discuss, the position of the II and X-ray tube has not only implications regarding magnification artifacts for the patient, but also important repercussions for X-ray exposure of the operator. Some recent biplane interventional radiology laboratories have been designed with the lateral gantry arranged more appropriately, with the II on the operator's side, which reduces the operator's radiation exposure; cardiac catheterization laboratories have not generally adopted this change.

Inside the X-ray tube, a current is passed through a filament in the cathode, thereby heating it and causing it to emit electrons. The current flowing from cathode to anode is expressed in milliamperes (mA). The cathode and anode are physically separated by a gap within a vacuum tube (i.e. the X-ray tube), and a voltage (expressed in kilovolts, $\mathrm{kV}$ ) is applied across them. Electrons released by the cathode are accelerated across the gap by the voltage applied across it and strike the target anode material, releasing their energy in the form of X-ray photons and heat. The number of electrons released per unit time by the cathode is proportional to the current passing through it, whereas the energy imparted to each electron as it exits the cathode and travels toward the anode is proportional to the voltage across the gap $(\mathrm{kV})$. Thus, the quantity of photons released when the electrons strike the anode is proportional to the $\mathrm{mA}$, whereas the maximal energy of the photons is dependent on the $\mathrm{kV}$. The role of $\mathrm{mA}$ and $\mathrm{kV}$ in image formation is discussed in the next section.

$\mathrm{X}$-ray photons emitted by the X-ray tube are focused into a relatively tight beam and exit in straight but diverging lines. The X-ray photons directed at the patient will have one of three possible fates: some of the photons will keep going straight through the patient without being altered in any way; others will be completely absorbed by the patient and terminate their path within the patient's tissues; and others still will interact with the tissues within the patient, and their energy will be partly lost within the tissues while their path will be deflected in another direction (the third fate is termed scatter). The relative proportion of photons that end in each fate has important implications for the risk to the patient as well as for the ultimate quality of the image produced. Dark areas on an X-ray film (or X-ray image receptor) are produced by X-ray photons that have reached the film to react with it, whereas white areas represent areas 
of void where X-rays have not reached the film. Gray areas represent parts of the film that have received some photons but not as many as dark areas. If all photons traversed through the patient without absorption or interaction with the tissues, then risk to the patient would be zero and the resulting image would be completely black. Conversely, if all X-rays were to be absorbed by the patient, then the film would be completely white, and the risk to the patient would be very high. Both of these scenarios would result in an image with no information gained about the patient's anatomy, however. Thus, some X-rays traversing the patient unchanged and some being absorbed by tissues are needed in order to obtain any meaningful image of the patient's anatomy. Consequently, absorbed rays are not merely a side effect of this imaging modality but an absolute requirement. Scattered rays, however, represent pure risk to the patient (because they still interact with tissues) with absolutely no imaging benefit. In fact, scattered rays are a major cause of image degradation and are also the single most important cause of X-ray exposure for the operator and catheterization laboratory personnel. Reduction of scatter is therefore of tremendous importance in improving image quality, reducing risk to the patient, and reducing exposure of personnel.

$\mathrm{X}$-rays that reach the II are converted into electrons once again by interacting with the input phosphor and photocathode; the electrons are then focused and accelerated onto the anode, where they strike and emit visible light. The light emitted is then focused and transmitted to a television monitor for viewing, or stored (using a digital video recorder or analog 35-mm cine film). An important feature of the imaging chain is the Automatic Exposure Control (AEC) feature that ensures relatively constant image brightness. AEC is accomplished by a feedback mechanism from the digital video processor to the X-ray generator; if conditions change such that fewer X-rays exit the patient (e.g., table has been moved such that a more radioopaque part of the body is now being imaged), then feedback to the X-ray generator will increase the quantity or intensity of X-rays in order to maintain equal image brightness. Alterations in beam characteristics by AEC are discussed in more detail below.

The catheterization laboratory is capable of several imaging modalities. The most frequently used is fluoroscopy. Fluoroscopy is used for live, real-time viewing and should provide sufficient image quality to view small guidewires. A high-dose fluoroscopy mode exists that might provide better penetration for obese patients, but its use should be strictly limited because of the increased radiation dose. Fluoroscopic imaging should be set to the lowest possible radiation settings consistent with a usable image quality. In the past, catheterization laboratories were equipped with continuous fluoroscopy; nowadays, variable-rate pulsed fluoroscopy is the standard. With pulsed fluoroscopy, the X-ray beam is pulsed at 30 or 15 pulses per second or fewer (the lower the pulse frequency the less the radiation dose, at the expense of a jerkier motion). Reviewing the previously acquired fluoroscopic image is possible with a feature known as "last-image hold"; the beam should be activated only when necessary to view a new live image of the heart. Furthermore, the foot should not be activating the fluoroscopy when the eyes are momentarily taken off the monitor (e.g., such as when looking at one's hands when introducing wires within a catheter, etc). An important reflex needs to be developed in those training in the use of fluoroscopy equipment: when the eye is not on the screen, the foot must not be on the pedal!

While fluoroscopic images can be stored permanently, their image quality is relatively poor, and images designed for permanent storage and review should be obtained in acquisition (cine) mode. Cine requires approximately 15 times more radiation per frame and should, therefore, be used sparingly [4]. Cine is always pulsed, and rates of 15 , 30 , or 60 frames per second are typically available in pediatric catheterization laboratories (whereas most adult studies are performed at 15 frames per second). Faster frame rates are necessary to view rapidly moving structures throughout the cardiac cycle (e.g., prosthetic valve leaflets) or in the setting of extremely high flow rates through a vascular bed (e.g., an arteriovenous fistula), and particularly if the patient is tachycardic. With both fluoroscopy and cine, limiting the time that the beam is turned on is crucial to limiting radiation dose.

Several equipment design challenges exist when considering the particular needs of pediatric cardiac catheterization laboratories. The first and most obvious difference is that adult laboratories are optimized to provide adequate image quality over a relatively narrow weight range (i.e. most adults weigh between 50 and $150 \mathrm{~kg}$ ), whereas the pediatric laboratory encounters patients who range from $<2 \mathrm{~kg}$ (the low-birthweight neonate) to the obese adolescent or young adult. The extremely wide range of weights poses a veritable challenge to equipment manufacturers, with the result being that a pediatric laboratory designed to accommodate all possible weights might not truly be optimal for any. For example, the II diameter in an adult laboratory will be adequate for imaging the heart in basically all adults. However, in the pediatric setting, cardiovascular imaging is rarely confined to the heart itself and might include imaging of any vascular structure within the thorax and abdomen (e.g., the central and distal pulmonary arteries, the central systemic veins, the aorta and its branches, etc.). Consequently, a given II diameter might be too small to visualize both lung fields simultaneously during pulmonary angiography in a large adolescent. Yet the same II might be so large as to completely cover the entire body of a premature infant, encumbering access to the patient and catheters. Larger IIs also limit the possible projection angles because of the risk of collisions between the frontal and lateral gantries, between the gantries and the table, or between the IIs and the patient.

Catheterizing small infants also poses additional risks to the operator because of the greater proximity of the operator to the primary beam and to the scatter (though a beneficial factor is that the beam intensity is lower in younger children). As a result, the use of hanging acrylic shields to protect the operator might not always be possible or practical during procedures on very small infants. 
Access via the jugular veins also renders more difficult the effective use of hanging shields.

\section{How X-ray beam characteristics affect image quality and radiation dose}

As discussed above, the quality and quantity of X-rays generated within the X-ray tube depend on a number of factors. Increasing the current applied through the filament within the cathode increases the quantity of electrons released by the cathode per unit time (expressed in $\mathrm{mA}$ ), which in turn increases the number of X-ray photons ultimately released when the electrons are caused to strike the anode. The current might range from $10 \mathrm{~mA}$ to $200 \mathrm{~mA}$ for pulsed fluoroscopy and from about $100 \mathrm{~mA}$ to $1,000 \mathrm{~mA}$ for cine angiography [5]. The $\mathrm{kV}$ represents the voltage difference between the anode and cathode. The higher the $\mathrm{kV}$, the faster the electrons are accelerated toward the anode, therefore releasing X-ray photons of higher energy upon collision with the anode. The typical setting of X-ray tube voltage for cardiac fluoroscopy is about $70 \mathrm{kV}$ [5]. Another characteristic of the X-ray tube that affects the X-ray beam is the pulse duration. In pulsed fluoroscopy (as well as in cine) the X-ray beam is pulsed on and off rapidly (e.g., at 30, 15 or 7.5 pulses per second), which results in lower total radiation dose than conventional continuous fluoroscopy. In pulsed fluoroscopy the time interval between pulses does not generate an image, and in order to reduce the impression of flicker of the image, the monitor displays the previously stored image until the subsequent pulse of X-rays generates a new image. Lowering the pulse frequency rate lowers the radiation dose proportionally. The duration of each pulse is also known as the exposure time, and is expressed in milliseconds (ms), with typical settings for pediatric cardiac fluoroscopy ranging from $1 \mathrm{~ms}$ to $4 \mathrm{~ms}$ per pulse [5].

Alterations in any of the characteristics of the X-ray beam ( $\mathrm{mA}, \mathrm{kV}$, and $\mathrm{ms}$ ) will result in specific changes to the image produced and also result in changes to the radiation dose. For example, increasing the $\mathrm{mA}$ will result in more X-ray photons (of the same energy range) in the beam, which in turn produces more darkening of the film. However, an increase in the $\mathrm{mA}$ results in a proportional increase in radiation dose. In order to illustrate the interaction of the various beam characteristics with the $\mathrm{AEC}$, let us consider the following example. During a procedure, if the frontal II is changed from a straight posteroanterior projection to a sharply angulated one (e.g., a cranially angulated left anterior oblique projection), the thickness that the X-ray beam will need to traverse will be greater. It is estimated that increasing the tissue thickness by $3-4 \mathrm{~cm}$ results in an image that is half as dense (a $3-\mathrm{cm}$ tissue thickness is considered one "half-value layer"). The AEC will, therefore, need to compensate in some fashion to correct the image density. Although doubling the pulse width (exposure time) would effectively result in a correction of the image density, this would also double the radiation dose per frame produced. Furthermore, longer pulse widths might result in images that exhibit motion artifact, rendering them less sharp overall. Doubling the $\mathrm{mA}$ would be another way to compensate for the increased tissue thickness, but this would also result in a doubling of the radiation dose, though it would also improve image contrast. The final option would be to increase the voltage by $6-10 \mathrm{kV}$. This would result in the production of higher energy X-ray photons, which would result in improved tissue penetration. Because fewer photons would be absorbed by the patient, this would actually result in a lower radiation dose to the patient compared to increasing the $\mathrm{mA}$ alone, at the cost of decreased image contrast. The purpose of the AEC system is to automatically adjust these parameters in order to maintain a relatively constant image density. AEC algorithms are different among different manufacturers, such that older units might only vary the $\mathrm{mA}$, while newer ones vary both the $\mathrm{mA}$ and $\mathrm{kV}$ to compensate for a change in image brightness.

Filtration of the X-ray beam is an important way to reduce the patient's radiation dose. Low-energy X-rays do not contribute to image formation and are instead absorbed in the patient's superficial tissues. For this reason, filtration of these photons (also known as "hardening" the beam) using a layer of aluminum (and in some units, copper is also used) at the exit port from the X-ray tube is beneficial, with minimal effect on image quality.

The X-ray beam is further altered by the use of collimators and partial-thickness filters. Collimators composed of lead shields are used to shape the beam at the exit port from the X-ray tube. The tighter the collimation, the less divergent the X-ray photons will be, which in turn reduces the amount of scatter. Reduction of scatter is extremely important because scattered radiation is the main cause of radiation exposure to the patient's body outside the field-of-view and to the personnel in the room. Scatter is also an important cause of image degradation; therefore, tight collimation on the region of interest also serves to improve image quality. Another benefit of tight collimation is that less volume of the patient's body is exposed to the primary beam (although reduction of scatter exposure to the patient's tissues is important, the primary beam is many times more intense than scattered rays). A qualification with respect to the benefits of tight collimation is in order, however: if collimation is extremely tight, part of the AEC sensor (typically located at the center of the image detector) might lie within the collimated region and will thus perceive reduced brightness. In such situations AEC compensation will result in an image that is excessively bright. Reducing the collimation somewhat will correct the problem.

Having discussed the characteristics of the beam at the X-ray tube, let us now turn our attention to the II. An important (and under-utilized) radiation reduction strategy in pediatric fluoroscopic imaging is the removal of the antiscatter grids. The grids are composed of lead strips designed to filter out scattered X-ray photons. Because the amount of scatter is greater with larger patients, the grids are most beneficial when used during studies on large patients. Conversely, their use in studies on young children 
results in relatively little improvement in image quality and, in fact, results in increased patient doses. This is because the grids also filter out some of the non-scattered rays, resulting in decreased image brightness; AEC compensation, therefore, leads to increased radiation dose in order to maintain image brightness at an acceptable level. Therefore, when used in studies on young children, the grids represent a cause of increased radiation exposure without significant benefit in image quality. Removal of the grids should be considered in these cases. One potential problem is that not all catheterization laboratories are equipped with grids that are easily removable. Even among our own laboratories at Texas Children's Hospital there is variability in the design of the grid attachments such that one unit has grids that can be removed in about 5 minutes with minimal effort, while another requires significant dismantling of the II unit and requires approximately 30 minutes to accomplish. Because pediatric catheterization laboratories care for patients with an extremely wide range of weights and sizes, the easy removal and replacement of antiscatter grids in between cases might not always be possible. However, a case that is expected to require a long fluoroscopy time in a small child should warrant the consideration of removal of the grid when this is feasible.

The age of the II also affects radiation dose because the efficiency of conversion of X-ray photons into light photons at the cesium iodide input phosphor decreases with time. The impaired efficiency with advancing age causes a compensatory increase in radiation dose. Furthermore, the image quality also degrades with advancing age. Competent biomedical engineers working with a radiation physicist must regularly evaluate catheterization laboratories for image quality and dose requirement.

An understanding of the various image magnification techniques is necessary to achieve optimal diagnostic accuracy while reducing radiation dose. The least costly method (in terms of radiation dose to the patient) of magnifying an image is the use of the "replay zoom" feature. This feature allows an image acquired in a nonmagnified fashion to be displayed on the monitors in a magnified view. Using the replay zoom feature produces an image that does not contain more information (pixels), but rather is simply displayed by replacing each pixel with a larger block on the screen. The end result is an image that is noticeably coarser and less pleasant to view. Reviewing an image in replay zoom mode can be helpful when making measurements of a small vessel, for example, because positioning the digital calipers on the edges of the vessel on the screen using the joystick is made easier when the target is amplified. However, vessels too small to accurately see in the standard view will not be viewed more easily in the replay zoom mode. A second type of magnification is called geometric magnification. This is accomplished by moving the II farther from the patient. As the II is moved farther from the patient, the shadows of the vascular structures cast upon it will be larger (analogous to moving a screen farther away from a television projector). The disadvantages of geometric magnification are significant.
First, the resulting image will be less bright and will thus prompt a compensatory increase in radiation dose by the AEC system. Second, the image will be affected by increased geometric blur, such that the edges of the vessels will be significantly less sharp. Last, the II, itself, actually helps to block some of the scattered rays to the personnel and to the operator, and moving the II away from the patient removes this protective effect. Overall, the use of geometric magnification is discouraged for all of these reasons.

The last type of magnification is called electronic magnification. This feature is particularly useful in pediatric patients because of the small anatomic structures that are being imaged. Electronic magnification is produced by reducing the field-of-view, such that a smaller surface area on the II is being exposed, with the resulting smaller exposed area being magnified to fill the entire visible screen. Most IIs have two or three "modes", or fields-of-view. For instance, a 9-inch II might have a 9-inch, a 7-inch, and a 5-inch mode (roughly equivalent to $23 \mathrm{~cm}, 17 \mathrm{~cm}$, and $13 \mathrm{~cm}$ ). Because a smaller area of the II receives the transmitted radiation, a loss of image brightness occurs, resulting in a compensatory increase in radiation dose. The increase in radiation is substantial, and thus electronic magnification should be used sparingly and only when truly necessary. The maximal increase in entrance exposure to the II is calculated to be the ratio of the area of the standard view over the area of the magnified view [3] (e.g., in going from a 9-inch mode to a 7 -inch mode, the exposure is increased by a factor of $9^{2} / 7^{2}=1.7$, while going from a 9 -inch mode to a 5 -inch mode increases the exposure by a factor of $9^{2} / 5^{2}=3.2$ ). This holds true if only the $\mathrm{mA}$ is increased when going to a magnified mode. With changes in $\mathrm{kV}$ also possible on some units, the actual increase in dose is sometimes significantly less than described by the above formula. Nonetheless, an increased dose is certain. However, the increased radiation cost to the patient is accompanied by an increase in image sharpness and resolution, thereby improving the visualization of small vascular structures. It is important to remember that an entire procedure need not be performed in a magnified mode: a critical angiogram can be acquired in magnified mode and the remainder of the procedure can then be carried out in the standard mode. Another disadvantage of overuse of the magnified mode is that excessive panning might be required to find adjacent structures that are out of the field-of-view. This is (unfortunately) commonly the case during coronary angiography, where the table might need to be moved (panned) during the angiogram to see the entire coronary tree. In the pediatric laboratory, where angiograms are almost always performed in biplane, and often in angulated views, resorting to panning to find the structures of interest during the course of an angiogram is almost never satisfactory because movement of the table in order to capture the desired region in one plane often results in loss of the region of interest in the other plane. Instead, a lesser degree of magnification should be used in order to avoid the need for panning altogether. As a general rule, the lowest acceptable level of magnification should be used that still allows sufficient diagnostic accuracy. 


\section{0 tactics for radiation dose reduction and image quality improvement}

The details of how to carry out a pediatric diagnostic or interventional cardiac catheterization are discussed in specialized texts [6-8], but a few points that impact radiation safety should be mentioned:

1. Diagnostic information should be obtained primarily non-invasively. A thorough history and physical examination should be obtained, followed by a complete echocardiogram in every patient prior to a cardiac catheterization. Determination of important anatomic variants (e.g., systemic venous anomalies) will help in the planning of the procedure (e.g., site of vascular access) and will serve to minimize the number of angiograms needed to clarify the anatomy. Furthermore, a prior understanding of the cardiovascular anatomy allows one to perform a more focused study directed at obtaining information that can uniquely be obtained by catheterization, which helps reduce radiation exposure. Only the necessary angiograms should be performed. One should avoid obtaining angiograms that provide redundant information already known from non-invasive studies "just because we're there".

2. Plan the angiographic projections ahead of time. Tables are available that list relatively standard angiographic views for best profiling some common defects $[6,9]$. However, children with rare or unusual anatomic variants need not be subject to a haphazard approach that consists of frequent angiograms guided by trial and error. This approach is costly to the patient in terms of contrast load, radiation dose, and procedure time. The angiographic projections needed to best demonstrate the anatomy can often be predicted based on non-invasive imaging. For instance, careful assessment of the location of a muscular ventricular septal defect by echocardiogram aids in predicting which angulated projections should best profile the defect at angiography. Or if an MR or CT study has been performed in a patient with branch pulmonary artery stenosis, reviewing these studies carefully can help predict how to best image the vessel angiographically with minimal foreshortening and minimal superimposition by surrounding vessels.

3. Place the patient in the isocenter and straight on the table. Having the patient in the isocenter facilitates keeping the heart at the center of the field despite changes in angulated views, without the need for prolonged fluoroscopy to adjust the patient's position with each change in angiographic projection. Another benefit of having the patient positioned correctly straight on the table is that certain cardiovascular structures can be reliably found with respect to skeletal and tracheobronchial landmarks (e.g., the fossa ovalis, the pulmonary arteries, the ductus arteriosus, etc) with minimal trial and error or wasted fluoroscopy.
4. Use the lowest acceptable frame rate during pulsed fluoroscopy and cine angiography. Always use pulsed fluoroscopy, never continuous fluoroscopy. Be prepared to change the frame rates frequently during a case depending on the type of structure that is being imaged (e.g., fast-moving vs. slow-moving; venous or arterial).

5. Do not use fluoroscopy to make changes to the patient/table position or collimators/shields. The patient should be moved first to the approximate desired location, then fluoroscopy should be used very briefly to check the position, followed by further patient adjustment, rather than using fluoroscopy constantly during patient movement. This is especially important when the patient needs to be moved by an assistant during the case (e.g., to reposition the arms in a different fashion). Do not apply fluoroscopy while the assistant is manipulating the patient! Many units have "virtual" markers that enable the positioning of collimators and partial thickness shields without the need for fluoroscopy by indicating their location on the screen. Even in units that do not have virtual markers, the collimators should still be moved first and checked for position with brief fluoroscopy; they should not be positioned during constant fluoroscopic visualization.

6. Remove unnecessary body parts (or instruments) from the field. A typical example of this is leaving the arms in the path of the beam. There is absolutely no reason for radiation burns to ever occur on the patient's arms, because the arms never belong in the field during routine cardiac studies. Indeed, the arms should never be visible on a cardiac study. Leaving the arms in the field not only results in needless radiation exposure to the arms, but also results in an overall increase in radiation exposure to all of the patient's tissues (and to the personnel) because the radioopaque arms drive the AEC to compensate with increased radiation output. The same can be said for the operator's hands: there is essentially no reason for the operator's hands to be visible on the screen at any time, and their presence in the path of the beam also drives up the radiation dose to patient and operator. Radioopaque instruments not only obscure the field of interest but also serve to drive up the radiation dose.

7. Use one angiogram to improve on subsequent angiographic projections. In cases where no other imaging studies have aided in the planning of angiography, it is acceptable to perform a first biplane angiogram in projections that are most likely to correspond to the given anatomy, or in the straight frontal and lateral projections. However, this first screening angiogram should be carefully scrutinized to determine how to plan subsequent angiograms (e.g., in the case of a coarctation of the aorta that is incompletely profiled, analysis of the lateral angiogram allows one to correct the subsequent angle of the frontal II, while analysis of the frontal angiogram 
allows one to correct the subsequent angle on the lateral II). In this fashion, the second set of angiograms should be able to perfectly profile the lesion, and a third angiogram should virtually never be necessary.

8. Always perform a test injection of a small amount of contrast material using fluoroscopy prior to acquiring an angiogram. This approach prevents, for example, the wasted angiogram that is taken with the catheter inadvertently wedged deeply in a vessel. Keep in mind that a few extra seconds of fluoroscopy and a tiny amount of contrast material are far less costly to the patient in contrast load and radiation exposure than a wasted angiogram. The fluoroscopy of the test injection can be useful to correct the angiographic projection prior to the actual angiogram, it can aid with determining the correct magnification mode (to prevent the need for panning if the magnification is too high), and it can even be stored and reviewed to help make these determinations.

9. Use the lowest acceptable magnification mode. As discussed, the replay zoom feature might be helpful in making measurements, at no radiation cost to the patient. Geometric magnification should not be used. Electronic magnification should be used sparingly, because of the substantial increase in radiation dose it requires. When in magnification mode for an angiogram, do not forget to return to the standard mode for the subsequent parts of the procedure when feasible. Remember that excessive magnification requires panning to search for the structure of interest, leading to a further waste of radiation dose.

10. Use collimators and partial thickness shields. Collimators are extremely beneficial overall in reducing the volume of tissue exposed to the primary beam and in reducing scatter; reducing scatter is, in turn, beneficial for reducing exposure to laboratory personnel and improves image contrast. Use of collimators and shields over radiolucent areas, such as the lung fields, improves exposure of the heart within the image. Certainly areas outside the body should not be visible at all (e.g., the space above the sternum on the lateral projection) and should be removed by optimal patient positioning and/or tight collimation. As a general rule, the collimators should be visible within the field, and studies should not be performed with the collimators wide open.

11. Center the region of interest correctly in the field. The center of the field has the least amount of image distortion; therefore, an angiogram should not intentionally be performed at the periphery of the field. Furthermore, bringing the region of interest to the center of the field allows for tighter collimation and less exposure of unnecessary patient tissues to the Xray beam. Last, the AEC sensor is typically at the center of the II, and thus optimal exposure of the structure of interest is best attained if it is brought into the center of the screen [1].
12. Keep the II as close to the patient as possible (and the $\mathrm{X}$-ray tube as far away as possible). The farther the II from the patient the higher the input doses and the greater the scatter to the laboratory personnel [4]. A distant II also results in geometric magnification, which introduces geometric blur. The second part of this heading is in parentheses because keeping the Xray tube as far away as possible, though recommended in general fluoroscopy, is not usually feasible or practical in pediatric cardiology. Although keeping the $\mathrm{X}$-ray tube far from the patient reduces radiation dose and is a very useful tactic in single plane fluoroscopy (in which case the table height would be set as high as possible), in biplane imaging the heart should be maintained at the isocenter.

13. Use angiographic projections that reduce operator exposure whenever possible. For example, the right anterior oblique projection moves the X-ray tube away from the operator, while the left anterior oblique projection moves it closer. The closer the operator to the X-ray tube, the closer he/she is to the surface of the patient that is emanating the highest amount of scatter.

14. Decrease beam-on time. This is one of the most important rules and has already been alluded to in point 4 above. Fluoroscopy must not be applied while discussing or contemplating the next maneuver. It is important to remember that if the eye is not on the screen, the foot should not be on the fluoroscopy pedal! Stored images, not live images, should be used for studying the case. During catheter exchanges, do not use fluoroscopy continuously, but rather check the wire position periodically with quick brief bursts of fluoroscopy.

15. Remove anti-scatter grids when catheterizing small children. As discussed above, this might not be feasible in some laboratories. When possible, however, a significant reduction in radiation dose is possible without compromising image quality.

16. Use X-ray stand position memory. Store useful projections in memory for rapidly returning to them when necessary without the need for fluoroscopy to verify position.

17. Use roadmap and overlay features. After a good quality angiogram is obtained, it can be used either in a side-by-side roadmap, or in some units it can be superimposed on a live fluoroscopy image that can be faded in or out of view as needed. These features allow vessels of interest to be found with minimal trial and error. They can assist with confirmation of wire position without the need for additional contrast injection.

18. Be familiar with your own laboratory equipment and features. Work with your biomedical engineering department, your radiation safety officer, a radiation physicist, and the manufacturer to regularly test and maintain the equipment in optimal working condition. Aging equipment will result in degradation of image quality and a need for higher radiation doses. Repair and/or replace aging equipment as required. 
19. Ensure protection of laboratory personnel. Before initiating fluoroscopy at the start of a procedure, ensure that everyone in the room is shielded. Ask everyone to move away from the patient during cine angiography. Make use of the inverse square law: doubling the distance from a point source reduces the radiation exposure to one-quarter. Operators must always make use of hanging acrylic shields. Other personnel should stand behind mobile shields when feasible. Insist on the proper use and storage of lead aprons: aprons that are improperly stored on hangers will be prone to developing cracks, which compromise their effectiveness. Insist on proper and consistent wearing of radiation badges.

20. Create a culture that strives toward radiation awareness and safety. Regularly discuss issues of radiation safety with nurses, technicians, and trainees. Include regular didactic seminars within a structured training program.

\section{Conclusion}

All who operate cardiac catheterization equipment must be trained and familiar with the basic principles of radiation safety. As the cardiac catheterization laboratory continues to expand its role in the diagnosis and treatment of congenital heart disease, the procedures are becoming increasingly intricate, and survivors of palliated complex congenital cardiac malformations are being repeatedly subjected to invasive procedures requiring ionizing radiation. With such tremendous ability to effectively treat such complex conditions comes an equal responsibility to use ionizing radiation responsibly. Attention to the simple rules of radiation safety and the use of tactics for planning and execution of cardiac catheterizations as outlined above should enable the pediatric cardiologist to produce highquality images at low radiation cost to the patient.

Acknowledgement I wish to acknowledge the helpful comments of Paul Murphy, Ph.D., during the preparation of this article.

\section{References}

1. Moore RJ (1985) The physics of cardiac angiography. Myrle Co. Enterprises, Riverside

2. Wagner LK, Archer BR (2000) Minimizing risks from fluoroscopic x-rays: bioeffects, instrumentation and examination. Partners in Radiation Management, The Woodlands

3. Limacher MC, Douglas PS, Germano G, et al (1998) ACC expert consensus document. Radiation safety in the practice of cardiology. American College of Cardiology. J Am Coll Cardiol 31:892-913

4. Hirshfeld JW Jr, Balter S, Brinker JA, et al (2004) ACCF/ AHA/HRS/SCAI clinical competence statement on physician knowledge to optimize patient safety and image quality in fluoroscopically guided invasive cardiovascular procedures. A report of the American College of Cardiology Foundation, American Heart Association, American College of Physicians Task Force on Clinical Competence and Training. J Am Coll Cardiol 44:2259-2282

5. Strauss KJ (1998) Cardiac catheterization equipment requirements: pediatric catheterization laboratory considerations. In: Nickoloff EL, Strauss KJ (eds) Categorical course in diagnostic radiology physics: cardiac catheterization imaging. Radiological Society of North America, pp 105-119

6. Freedom RM, Mawson JB, Yoo SJ, et al (1997) Congenital heart disease: textbook of angiocardiography. Futura, Armonk

7. Mullins CE (2006) Cardiac catheterization in congenital heart disease: pediatric and adult. Blackwell/Futura, Malden

8. Lock JE, Keane JF, Perry SB (1999) Diagnostic and interventional catheterization in congenital heart disease. Springer, Berlin Heidelberg New York

9. Nihill MR (1998) Catheterization and angiography. In: Garson A, Bricker JT, Fisher DJ, Neish SR (eds) The science and practice of pediatric cardiology. Williams \& Wilkins, Baltimore, pp 995-1019 